\title{
Prevalence of Mild Behavioral Impairment and Risk of Dementia in a Psychiatric Outpatient Clinic
}

\author{
Teruyuki Matsuoka ${ }^{\mathrm{a}, *}$, Zahinoor Ismail ${ }^{\mathrm{b}}$ and Jin Narumoto ${ }^{\mathrm{a}}$ \\ ${ }^{a}$ Department of Psychiatry, Graduate School of Medical Science, Kyoto Prefectural University of Medicine, \\ Kyoto, Japan \\ ${ }^{\mathrm{b}}$ Departments of Psychiatry, Clinical Neurosciences, and Community Health Sciences, Hotchkiss Brain Institute \\ and O'Brien Institute for Public Health, University of Calgary, Calgary, Canada
}

Handling Associate Editor: Carlo Abbate

Accepted 9 May 2019

\begin{abstract}
.
Background: Mild behavioral impairment (MBI) has been proposed as risk factor for dementia, and for some, an early manifestation of dementia.

Objective: We examined the prevalence of MBI in the psychiatric outpatient clinic, and compared the incidence of dementia in MBI with that in other psychiatric diseases.

Methods: Retrospective chart review was conducted in 2,853 consecutive outpatients over the age of 50. MBI was diagnosed according to the International Society to Advance Alzheimer's Research and Treatment research diagnostic criteria. The incidence rate of dementia was examined in the patients who were followed up for at least 1 month. Kaplan-Meier survival analyses and Cox proportional hazards regression models were performed to compare the time to onset of dementia between MBI and other psychiatric diseases.

Results: The prevalence of MBI was 3.5\% and the incidence of dementia was 30.7 cases per 1000 person-years. The hazard ratio (HR) for dementia was higher for MBI than other psychiatric diseases (HR: 8.07, 95\% confidence interval: 4.34-15.03, $p<0.001$ ). In MCI patients, the cumulative survival in MCI with affective dysregulation tended to be lower than that in MCI without $(p=0.090)$.

Conclusions: Psychiatric outpatients often meet MBI criteria. MBI, especially the affective dysregulation domain, increases the risk of dementia in this psychiatric outpatient population. Since late-onset psychiatric and behavioral symptoms may be prodromal symptoms of dementia in some, careful observation is needed, and psychiatric clinicians should keep prodromal dementia on their differential diagnosis when assessing those with new onset psychiatric symptomatology in older adults.
\end{abstract}

Keywords: Dementia, depression, mild behavioral impairment, mild cognitive impairment, neuropsychiatric symptoms, sleep disorder, subjective cognitive decline

\footnotetext{
${ }^{*}$ Correspondence to: Teruyuki Matsuoka, Department of Psychiatry, Graduate School of Medical Science, Kyoto Prefectural University of Medicine, 465 Kajii-cho, Kawaramachi-Hirokoji, Kamigyo-ku, Kyoto 602-8566, Japan. Tel.: +81 75 2515612; Fax: +81 75 2515839; E-mail: tmms2004@koto.kpu-m.ac.jp.
}

\section{INTRODUCTION}

Neuropsychiatric symptoms (NPS) are common in the older population. A previous community based study reported that $38.9 \%$ of community-dwelling older adults had NPS [1], with the prevalence 
increasing as cognitive function declined. The rates ranged from $30.8 \%$ in the cognitively normal population to $80 \%$ in those with dementia [1].

Increasingly, NPS have been considered as a risk factor for all-cause dementia or even early symptoms of dementia. Psychiatric symptoms, including sleep disturbance [2,3] and depression [4, 5], are risk factors of dementia. Sleep disturbance might contribute to dysfunction of amyloid clearance, and then contribute to the development of Alzheimer's disease (AD) [6,7]. Affective neuropsychiatric symptoms including depressive symptoms are predictors of dementia even in those with normal cognition and subjective cognitive decline (SCD) [8]. While the annual progression rate of dementia in patients with mild cognitive impairment (MCI) is approximately $12 \%$ [9], the progression rate in MCI patients with NPS is estimated at 25\% [10]. Those with NPS have a 3-fold greater risk of dementia than those without NPS [1]. NPS, especially psychosis, agitation, and aggression, were detected as predictors of progression to severe dementia and death [11]. Moreover, NPS are often identified as early symptoms of dementia [12], but these patients often start out in psychiatric care.

Mild behavioral impairment (MBI) has been proposed as a risk factor for dementia and for some may be the index manifestation of dementia. MBI without overt cognitive symptoms had initially been considered as the prodromal stage of frontotemporal dementia [13]. However, the International Society to Advance Alzheimer's Research and Treatment (ISTAART) proposed newer research diagnostic criteria for MBI as an at-risk state for all cause dementia, given that NPS are common in all dementias [14]. MBI is characterized by later life emergent and sustained NPS in the following domains: 1) decreased motivation, interest and drive (apathy); 2) emotional or affective dysregulation (mood and anxiety symptoms); 3) impulse dyscontrol (agitation, aggression, and abnormal reward salience); 4) social inappropriateness (impaired social cognition); and 5) abnormal thoughts and perception (psychotic symptoms). Importantly, the ISTAART MBI criteria made explicit the relationship between $\mathrm{MBI}$ and MCI in that MBI could occur in advance of, in concert with, or following MCI. Thus, MBI reflects the neurobehavioral axis of pre-dementia risk states, as a complement to the neurocognitive axis represented by SCD and MCI. A recently published 5-year longitudinal study demonstrated a higher rate of incident dementia in MBI compared to late life psychiatric illness, demonstrating the clinical significance of the MBI syndrome, and the importance in distinguishing it from psychiatric illness recurring in late life [15]. Additionally, in a large community sample of cognitively unimpaired participants, MBI demonstrated an associated with faster decline in attention and working memory [16]. Recent genetic evidence has also demonstrated a common etiology between $\mathrm{MBI}$ and $\mathrm{AD}$, suggesting that neurodegeneration may contribute to the emergence of neuropsychiatric symptoms, as it does with emergent neurocognitive symptoms [17]. Case ascertainment for MBI has been operationalized with the MBI checklist (MBIC, available at http://www.MBItest.org) [18], a rating scale that accurately reflects the MBI criteria with respect to the MBI domains, requirement of symptoms to be of 6 months duration (thus decreasing false positives from reactive states and fluctuating symptoms), explicitly mandating that symptoms be emergent in later life, and using language targeted toward community dwelling functionally independent older adults (as opposed to dementia-centric language). Using the MBI-C in a primary care population, MBI prevalence was determined to be $5.8 \%$ in SCD [19], and $14.2 \%$ in MCI [20]. As the MBI$\mathrm{C}$ is a very new instrument, other groups have used the neuropsychiatric inventory (NPI) to estimate MBI frequency by mapping NPI items onto MBI domains. The drawback of this approach is the relatively short reference range of the NPI of 1 month, increasing the possibility of false positives. Accordingly, the prevalence of MBI using this approach has been substantially higher. In a community population, MBI prevalence was $43.1 \%$ in SCD, and $48.9 \%$ in MCI [21]. In a cognitive neurology clinic population, the prevalence was higher still at $76.5 \%$ in SCD, and $85.3 \%$ in MCI, and was associated with greater caregiver burden in both groups [22]. What is not known is the prevalence of MBI in a specialty psychiatric clinic. Thus, the aim of this study was to examine the prevalence of MBI in the psychiatric outpatient clinic, and compare the incidence of dementia in MBI with that in other conditions, especially MCI, SCD, sleep disorder, and depression.

\section{METHODS}

\section{Subjects}

We conducted a retrospective chart review of 2,853 consecutive outpatients over the age of 50 (1,076 men and 1,777 women; mean age \pm standard deviation, 
$68.9 \pm 11.1$ years old) who were seen at the department of Psychiatry and the Center for Diagnosis of Dementia, Kyoto Prefectural University of Medicine, between April 2009 and March 2016. Since psychiatrists are in charge of the Center for Diagnosis of Dementia in our hospital, our psychiatric outpatient clinic includes the outpatient clinic of the center. Therefore, the older people with NPS are often referred to our psychiatric outpatient clinic. Moreover, MCI or SCD without NPS were sometimes referred to our psychiatric outpatient clinic because some psychiatrists perform the differential diagnosis of dementia. We investigated the prevalence and features of MBI. One rater (T.M., board certified specialist of the Japanese Society of Psychiatry and Neurology and the Japanese Psychogeriatric Society) retrospectively diagnosed MBI in accordance with the ISTAART research diagnostic criteria [14]. In patients without dementia who were followed up for at least 1 month, we examined the incidence rate of dementia at December 2017. The diagnosis was made by psychiatrists, according to the International Classification of Disease (ICD-10). Dementia with Lewy bodies (DLB) and MCI were diagnosed using the DLB consensus criteria [23] and Petersen criteria [9], respectively. In the current study, MCI or SCD without MBI was defined as the patients met the criteria of MCI or SCD, but not the criteria of MBI. According to the previous studies [22, 24], SCD was defined as having subjective persistent cognitive complaints but not meeting the criteria of MCI or dementia. Rapid eye movement sleep behavior disorder (RBD) was diagnosed only on the interview of clinical symptoms, without polysomnography. All data were coded and registered anonymously. The Ethics Committee of Kyoto Prefectural University of Medicine approved this retrospective study. We provided patients with information on the right to refuse the study, presenting it both in the waiting room of the outpatient clinic and on the institutional homepage. When participants did not agree with the contents of study, they were excluded from the study.

\section{Statistical analyses}

We used the Chi square test to exam the differences between genders. The comparison of duration between diagnosis and dementia onset was performed using $t$-test. Kaplan-Meier survival analyses with $\log$ rank tests were performed to compare the time to onset of dementia between MBI, MCI without
MBI, SCD without MBI, sleep disorder, depressive episode, and other diagnoses excluded these diagnoses. Cox proportional hazards regression models with a forced entry method were also conducted to estimate the hazard ratio (HR) of dementia. The independent variables included age, gender, and diagnosis. In the diagnosis, the HR for MBI, MCI without MBI, SCD without MBI, sleep disorder, and depressive episode were estimated compared to other diagnoses excluding these diagnoses. To compare each MBI domain, Kaplan-Meier survival analyses with log rank tests and Cox proportional hazards regression models with a forced entry method were also performed in MCI patients only. In these analyses, MCI with each MBI domain was compared with MCI without each MBI domain. The independent variables included age, gender, and each MBI domain. Data were analyzed using SPSS 22 (IBM Corp., Armonk, NY, USA), and $p<0.05$ was considered statistically significant in these tests.

\section{RESULTS}

\section{Characteristics of subjects}

In all patients, the F4 category (neurotic, stressrelated, and somatoform disorders; $n=905$ ) was the most common diagnosis, followed by F0 (organic, including symptomatic, mental disorders; $n=878$ ), F3 (mood disorders; $n=516$ ), and F2 (schizophrenia, schizotypal, and delusional disorders; $n=207) .100$ out of 2,853 psychiatric outpatients (3.5\%) actually met the criteria for MBI. Among 100 patients with MBI, 90 patients also met the criteria of MCI, and 10 patients were also considered as SCD. Since the total number of MCI and SCD patients were 180 and 51 , respectively, $50.0 \%$ of MCI patients and $19.6 \%$ of SCD patients met the MBI criteria. MBI patients consisted of 25 men and 75 women, and the mean age was $76.3 \pm 7.2$ years old. MBI was more common in woman than in men $(75 / 1777(4.2 \%)$ versus $25 / 1076$ $(2.3 \%), p=0.008)$.

For MBI domains, affective dysregulation was the most common (64\%), followed by abnormal perception or thought content (21\%), impulse dyscontrol $(12 \%)$, decreased motivation (11\%), and social inappropriateness $(1 \%)$. In each domain, there was a significant difference only in abnormal perception or thought content between women and men (18/1777 $(1.0 \%)$ versus $3 / 1076(0.3 \%), p=0.026)$. 
Table 1

The incidence rate of dementia at each disease

\begin{tabular}{lcc}
\hline Disease & $\begin{array}{c}\text { Dementia incidence } \\
\text { (cases per 1000 } \\
\text { person-year } \\
\text { of follow up) }\end{array}$ & $\begin{array}{c}\text { Duration between } \\
\text { diagnosis and } \\
\text { dementia } \\
\text { onset (month) }\end{array}$ \\
\hline Total & 30.7 & $18.0 \pm 14.2$ \\
MBI & 236.5 & $17.0 \pm 12.6$ \\
MCI without MBI & 230.4 & $15.8 \pm 12.4$ \\
Delirium & 149.3 & 26 \\
SCD without MBI & 107.9 & $16.0 \pm 5.6$ \\
Delusional disorder & 88.7 & $37.2 \pm 19.0$ \\
Other nonorganic psychotic disorder & 38.8 & 40 \\
Anxiety disorder & 27.1 & $10.2 \pm 7.0$ \\
Depressive episode & 16.2 & $18.8 \pm 18.9$ \\
Adjustment disorder & 12.3 & $7.0 \pm 5.6$ \\
Nonorganic sleep disorder & 7.5 & 32 \\
Somatoform disorder & 5.9 & $22.5 \pm 12.0$ \\
\hline
\end{tabular}

MBI, mild behavioral impairment; MCI, mild cognitive impairment; SCD, subjective cognitive decline.

\section{Incidence of dementia}

In 2,853 total outpatients, 2,218 patients did not have dementia at the first visit. 1,329 out of 2,218 patients were followed up for at least 1 month. The follow-up period of these patients were $22.7 \pm 23.9$ months (range: 1 to 104 months). 77 out of 1,329 $(5.8 \%)$ patients developed dementia, mostly AD $(66 / 77(85.7 \%))$. In the 77 patients who developed dementia, the most common diagnosis at the first visit was MBI $(n=29)$, followed by MCI without MBI $(n=17)$, depressive episode $(n=9)$, anxiety disorder $(n=5)$, delusional disorder $(n=5)$, adjustment disorder $(n=4)$, SCD without MBI $(n=3)$, somatoform disorder $(n=2)$, nonorganic sleep disorder $(n=1)$, delirium $(n=1)$, and other nonorganic psychotic disorder $(n=1)$. Table 1 showed the incidence rate of dementia at each disease. Three out of 73 patients with sleep disorder met the criteria of RBD, and 1 out of three patients with RBD developed DLB (incidence rate of dementia: 148.4 cases per 1000 person-years of follow up). The duration between diagnosis and dementia onset in adjustment disorder and anxiety disorder was relatively shorter than that in MBI, although there are no significant differences ( $p=0.130$ and $p=0.249$, respectively).

Figure 1 shows the results of Kaplan-Meier survival analysis with log rank tests. There were significant differences between MBI and sleep disor$\operatorname{der}(p<0.001)$, between MBI and depressive episode $(p<0.001)$, and between MBI and other diagnoses $(p<0.001)$, while there were no differences between MBI and MCI without MBI ( $p=0.987)$, and between MBI and SCD without MBI $(p=0.283)$.

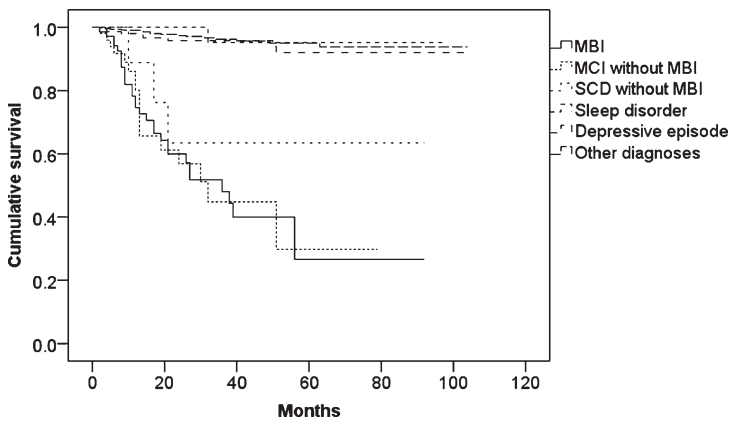

Fig. 1. The results of Kaplan-Meier survival analysis in MBI, MCI without MBI, SCD without MBI, nonorganic sleep disorder, depressive episode, and other diagnoses excluded these diagnoses.

Table 2

Results of the cox proportional hazards regression models with a forced entry method. Hazard ratio of dementia in MBI, MCI without MBI, SCD without MBI, sleep disorder, and depressive episode were estimated compared to other diagnoses excluded these diagnoses

\begin{tabular}{lccc}
\hline Variable & $\begin{array}{c}\text { Hazard } \\
\text { ratio }\end{array}$ & $\begin{array}{c}95 \% \text { confidence } \\
\text { interval }\end{array}$ & $p$ value \\
\hline MBI & 8.07 & $4.34-15.03$ & $<0.001$ \\
MCI without MBI & 7.05 & $3.50-14.21$ & $<0.001$ \\
SCD without MBI & 6.81 & $1.99-23.27$ & 0.002 \\
Sleep disorder & 0.71 & $0.09-5.41$ & 0.744 \\
Depressive episode & 1.40 & $0.63-3.13$ & 0.407 \\
Age & 1.10 & $1.07-1.13$ & $<0.001$ \\
Male & 0.84 & $0.48-1.46$ & 0.536 \\
\hline
\end{tabular}

In the Cox proportional hazards regression models with a forced entry method, MBI, MCI without MBI, SCD without MBI, and age were significant independent variables. The HR in MBI was the highest (Table 2). 
Among 180 MCI patients, 131 patients were followed up for at least 1 month. Kaplan-Meier survival analyses with log rank tests showed a significant difference between MCI with and without abnormal perception or thought content $(p=0.045)$ (Fig. 2). The cumulative survival between MCI with and without affective dysregulation tended to be different $(p=0.090)$. In the Cox proportional hazards regression models with a forced entry method, the HR in MCI with abnormal perception or thought content MBI was 0.307 (95\% confidence interval: $0.094-0.999, p=0.050$ ), and the HR in MCI with affective dysregulation was 1.646 (95\% confidence interval: 0.903-2.999, $p=0.104)$.

\section{DISCUSSION}

In the psychiatric outpatient clinic, the prevalence of MBI was 3.5\% and the incidence rate of dementia was 30.7 cases per 1000 person-years. MBI, MCI without MBI, and SCD without MBI increased the risk of dementia, while sleep disorder and depressive episode did not. In the MCI patients, those with affective dysregulation tended to develop dementia with a hazard ratio of 1.646 compared to those without. Therefore, MBI, especially affective dysregulation domain, might be associated with dementia.

While the prevalence of MBI was $3.5 \%$ in all outpatients, the prevalence was $50.0 \%$ in MCI and $19.6 \%$ in SCD patients. The prevalence of MBI in our psychiatric clinic was relatively low compared with previous studies in a neurology clinic, which likely overestimated MBI prevalence due to requiring only one-month symptom duration as per the Neuropsychiatric Inventory [21, 22]. Further, the difference of clinical setting (psychiatric versus neurology clinic) may affect the results. Psychiatrists might tend to diagnose the late onset psychiatric symptoms as psychiatric disease. This lower prevalence estimate may also be due to the retrospective study design and that those with SCD alone are not typically referred to a psychiatric clinic. However, when we look at our prevalence in SCD and MCI, MBI prevalence in our psychiatric clinic was higher than in the primary care population, which used the MBI checklist for case ascertainment $[19,20]$. Given that the prevalence of NPS is higher in clinical versus community samples [25] and in clinical settings the specialty clinic prevalence is higher than in primary care, the greater MBI prevalence in SCD and MCI in the current study and previous studies $[21,22]$ versus the previously published Spanish primary care sample may be expected. However, in the recent studies using MBI checklist $[19,20]$, MBI checklist was administered by phone to an informant, which might affect case detection compared to our method of retrospective chart review.

For the MBI domains, the results of the current study and previous studies $[21,22]$ indicate that affective dysregulation is the most common domain in MBI irrespective of sample setting or ascertainment method, while the prevalence of other domains was inconsistent. The difference in prevalence of the other MBI domains might be caused by the different sample and setting or different ascertainment methods. First, as the previous study pointed out, the patients with NPS are more likely to attend the clinic [22]. Given the fact that our study was conducted in a
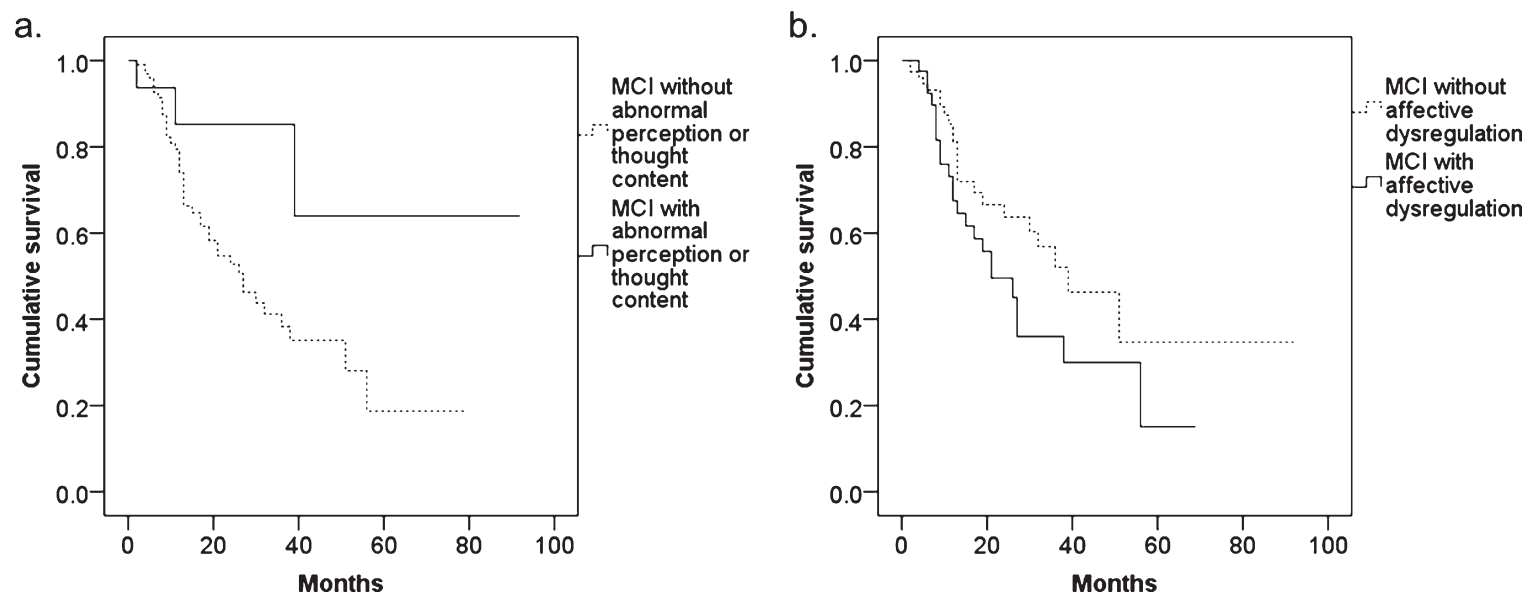

Fig. 2. The results of Kaplan-Meier survival analysis in MCI patients with and without (a) abnormal perception or thought content and (b) affective dysregulation. 
psychiatry outpatient clinic (as opposed to a cognitive neurology clinic), the prevalence of abnormal perception or thought content might be higher still, as these patients may be preferentially referred to psychiatry versus neurology. Second, when measured with a rating scale, impulse dyscontrol is likely more frequent than in a retrospective chart review like ours. This may reflect a lack of charting or observation about impulse dyscontrol, or the assumption in a psychiatric clinic that this symptom is subsumed under other psychiatric conditions.

Gender differences in MBI have been previously reported. MBI, especially decreased motivation and impulse dyscontrol, is more common in men than in women [21]. In the current study, the prevalence of MBI in women was more common than in men, especially in the abnormal perception or thought content domain. These results suggests that decreased motivation and impulse dyscontrol domains were more common symptoms in men, and abnormal perception or thought content domain were more common in women.

Dementia incidence in the current study was 30.7 cases per 1000 person-years. This is similar to a previous study reporting that dementia incidence was 32.5 cases per 1000 person-years in people over 65 years old from the general population [26]. Most patients developed AD in the current study. The dementia incidence and HR of dementia was highest in MBI, although the incidence was close to that for MCI without MBI. While diagnosed depression did not increase the risk of dementia in the current study, the affective dysregulation domain of MBI did increase the risk of dementia. Our results are consistent with recent literature. According to previous reviews, depression in early life might be a risk factor for dementia, and late life onset of depression might be a prodromal symptom [4, 27]. Evidence from recent large longitudinal cohorts also supports the importance of age of onset of depressive symptoms [12, 27-29]. These studies describe the age of onset of depressive symptomatology as an important component of their dementia risk. For some, incident depressive symptoms were diagnosed as depression in the traditional sense, but were in fact prodromal symptoms of dementia. Moreover, in the current study, anxiety disorder had shorter duration between diagnosis and the onset of dementia compared to MBI. It is possible that the symptoms of an anxiety disorder, diagnosed through a psychiatric lens in a psychiatric clinic, were in fact the prodromal manifestations of dementia. Anxiety (part of the
MBI affective dysregulation domain) has been associated with striatal amyloid- $\beta$ in cognitive normals [30], suggesting that the underlying AD neuropathological process may drive this symptom in some. Case reports have also described emergent psychiatric symptoms being given psychiatric diagnoses in advance of dementia declaring itself [31, 32]. Further, in a retrospective review of dementia patients in a cognitive neurology clinic, $28.2 \%$ were initially given a psychiatric diagnosis, with these symptoms ultimately representing prodromal dementia as opposed to formal psychiatric conditions [33]. While 52.2\% of the patients in this study with initial psychiatric diagnoses developed frontotemporal dementia (FTD), 23.1\% developed AD, suggesting that early emergence of psychiatric symptoms is not simply relegated to FTD, but also relevant to other dementias including $\mathrm{AD}$ [33]. Overall, our results are consistent with previous literature suggesting that for some, late onset psychiatric symptomatology (especially mood and anxiety symptoms) can be given a psychiatric diagnosis in primary and specialty care, warranting greater vigilance in these settings for emergent dementia.

Interestingly, the risk of dementia in MCI with abnormal perception or thought content was lower than that in MCI without. This might be the result of challenges with differential diagnoses. In the current study, the prevalence of abnormal perception or thought content domain was higher than those of previous studies (as might be expected in a psychiatric clinic) [21, 22]. Since patients with late-onset delusional disorder have impairment of cognition including divided attention and visuo-perception with planning and organization [34], patients with lateonset delusional disorder might be diagnosed as MCI. On the other hand, the dementia incidence in delusional disorder was relatively higher than other psychiatric diseases. It is speculated that some of those with abnormal perception or thought content were classified as delusional disorder instead of the delusions as being part of the MBI. As a previous study pointed out, late onset psychosis (LOP) might be misdiagnosed as psychiatric disease, such as delusional disorder, when it might be better categorized as the emerging psychosis of a neurodegenerative disease [12]. LOP is often seen in older people. The main etiology of LOP is dementia, delusional disorder, schizophrenia, and depression [35]. MCI patients with delusions might be diagnosed as delusional disorder in ICD-10, especially when the delusion is relatively strong. It is often difficult to distinguish 
between NPS in neurodegenerative disease and lateonset psychiatric disease. The etiology of LOP is various, and then the careful observation is needed for patients with LOP.

Despite a very small proportion of our subjects having SCD, SCD without MBI increased the risk of dementia in the current study, while all $6 \mathrm{SCD}$ patients with MBI did not develop dementia during the follow-up period. This is a finding that may or may not be explained sample size issues. While SCD has been proposed as the first symptom of $\mathrm{AD}$ [24], and increasing the risk of $\mathrm{AD}$ [36], these patients are generally not referred to psychiatry clinics unless comorbid with psychiatric symptomatology. In Japan, people with subjective cognitive complaints are sometimes seen at psychiatric outpatient clinic because they are worried about dementia. This worry could be potentially mood and anxiety symptoms of MBI. As previous review pointed out, subjective cognitive complaints are affected by depression [37]. One can speculate that the cognitive complaints may have been part of a depressive syndrome and without information on the age of onset of the depressive syndrome, it is difficult to determine if it is part of MBI or rather subclinical depression. Further study is necessary to elucidate the relationship between SCD, depressive symptoms, and dementia, bearing in mind that this is not generally the presenting complaint to general psychiatric clinics.

Sleep disorder was not a risk factor for dementia in the current study, although previous studies have implicated sleep disturbance as risk factor [2,3]. Sleep inadequacy and day-time sleepiness increased the risk of dementia about 1.2-fold in non-demented community-dwelling elderly people [2]. A prospective cohort study in a Japanese community identified short and long daily sleep duration and hypnotic use as the risk factors of dementia [3]. The inconsistency might be caused by the method of the current study. The current study was retrospective study, and the follow-up period was relatively short. While the mean follow-up period of these previous studies was 3 to 8.8 years [2,3], the mean period of the current study was 1.9 years. Among 73 patients with sleep disorder in the current study, one patient with RBD developed DLB after 2.7 years. Since RBD is included in the core clinical features of DLB [38], RBD might be presented as the prodromal symptoms of DLB.

There were some limitations in the current study. First, the duration of follow-up was relatively short for some participants, which is especially impactful in determining dementia incidence for the SCD group, where typically time to dementia is significantly longer than it is for MCI. Therefore, it was not elucidated whether MBI reflects prodromal symptoms or a risk factor of dementia in this group. Second, in the comparison of duration between diagnosis and dementia onset, there were no significant differences between MBI and other psychiatric diseases. These findings may be due to retrospective methodological limitations and variable follow up times. Finally, the information from chart and assessments of cognitive function and NPS might be insufficient because of the retrospective design. NPS were not always assessed using the checklist such as Neuropsychiatric Inventory. As the previous study pointed out [12], late onset psychiatric symptoms might be misdiagnosed as psychiatric disease. Hence, the prevalence of MBI and the incidence rate of dementia might be inaccurate. A prospective study using the MBI checklist is needed.

In conclusion, psychiatric outpatients often meet the criteria of MBI, and those with later life onset of psychiatric symptoms can be referred to psychiatric clinics and given psychiatric diagnoses. In this group, MBI, especially the affective dysregulation domain, increases the risk of AD. Since the late-onset psychotic and behavioral symptoms may be prodromal symptoms of dementia in some, careful observation is needed, and clinicians in psychiatric clinics should keep prodromal dementia on their differential diagnosis, when assessing those with new onset psychiatric symptomatology in older adults.

\section{ACKNOWLEDGMENTS}

This research was supported by a Grant-in-Aid for Young Scientists (B) Grant number 17K16392.

Authors' disclosures available online (https:// www.j-alz.com/manuscript-disclosures/19-0278r1).

\section{REFERENCES}

[1] Mortby ME, Burns R, Eramu dugolla R, Ismail Z, Anstey KJ (2017) Neuropsychiatric symptoms and cognitive impairment: Understanding the importance of co-morbid symptoms. J Alzheimers Dis 59, 141-153.

[2] Tsapanou A, Gu Y, Manly J, Schupf N, Tang MX, Zimmerman M, Scarmeas N, Stern Y (2015) Daytime sleepiness and sleep inadequacy as risk factors for dementia. Dement Geriatr Cogn Dis Extra 5, 286-295.

[3] Ohara T, Honda T, Hata J, Yoshida D, Mukai N, Hirakawa Y, Shibata M, Kishimoto H, Kitazono T, Kanba S, Ninomiya $\mathrm{T}$ (2018) Association between daily sleep duration and risk of dementia and mortality in a Japanese community. J Am Geriatr Soc 66, 1911-1918. 
[4] Bennett S, Thomas AJ (2014) Depression and dementia: Cause, consequence or coincidence? Maturitas 79, 184-190.

[5] Burke SL, Maramaldi P, Cadet T, Kukull W (2018) Decreasing hazards of Alzheimer's disease with the use of antidepressants: Mitigating the risk of depression and apolipoprotein E. Int J Geriatr Psychiatry 33, 200-211.

[6] Yulug B, Hanoglu L, Kilic E (2017) Does sleep disturbance affect the amyloid clearance mechanisms in Alzheimer's disease? Psychiatry Clin Neurosci 71, 673-677.

[7] Matsuoka T, Imai A, Fujimoto H, Kato Y, Shibata K, Nakamura K, Yokota H, Yamada K, Narumoto J (2018) Neural correlates of sleep disturbance in Alzheimer's disease: Role of the precuneus in sleep disturbance. J Alzheimers Dis $\mathbf{6 3}$, 957-964.

[8] Donovan NJ, Amariglio RE, Zoller AS, Rudel RK, GomezIsla T, Blacker D, Hyman BT, Locascio JJ, Johnson KA, Sperling RA, Marshall GA, Rentz DM (2014) Subjective cognitive concerns and neuropsychiatric predictors of progression to the early clinical stages of Alzheimer disease. Am J Geriatr Psychiatry 22, 1642-1651.

[9] Petersen RC (2004) Mild cognitive impairment as a diagnostic entity. J Intern Med 256, 183-194.

[10] Rosenberg PB, Mielke MM, Appleby BS, Oh ES, Geda YE, Lyketsos CG (2013) The association of neuropsychiatric symptoms in MCI with incident dementia and Alzheimer disease. Am J Geriatr Psychiatry 21, 685-695.

[11] Peters ME, Schwartz S, Han D, Rabins PV, Steinberg M, Tschanz JT, Lyketsos CG (2015) Neuropsychiatric symptoms as predictors of progression to severe Alzheimer's dementia and death: The Cache County Dementia Progression Study. Am J Psychiatry 172, 460-465.

[12] Tapiainen V, Hartikainen S, Taipale H, Tiihonen J, Tolppanen AM (2017) Hospital-treated mental and behavioral disorders and risk of Alzheimer's disease: A nationwide nested case-control study. Eur Psychiatry 43, 92-98.

[13] Taragano FE, Allegri RF, Krupitzki H, Sarasola DR, Serrano CM, Lon L, Lyketsos CG (2009) Mild behavioral impairment and risk of dementia: A prospective cohort study of 358 patients. J Clin Psychiatry 70, 584-592.

[14] Ismail Z, Smith EE, Geda Y, Sultzer D, Brodaty H, Smith G, Aguera-Ortiz L, Sweet R, Miller D, Lyketsos CG, ISTAART Neuropsychiatric Symptoms Professional Interest Area (2016) Neuropsychiatric symptoms as early manifestations of emergent dementia: Provisional diagnostic criteria for mild behavioral impairment. Alzheimers Dement 12, 195-202.

[15] Taragano FE, Allegri RF, Heisecke SL, Martelli MI, Feldman ML, Sanchez V, Garcia VA, Tufro G, Castro DM, Leguizamon PP, Guelar V, Ruotolo E, Zegarra C, Dillon C (2018) Risk of conversion to dementia in a mild behavioral impairment group compared to a psychiatric group and to a mild cognitive impairment group. J Alzheimers Dis $\mathbf{6 2}$, 227-238.

[16] Creese B, Brooker H, Ismail Z, Wesnes KA, Hampshire A, Khan Z, Megalogeni M, Corbett A, Aarsland D, Ballard C (2019) Mild behavioral impairment as a marker of cognitive decline in cognitively normal older adults. Am J Geriatr Psychiatry, doi: 10.1016/j.jagp.2019.01.215

[17] Andrews SJ, Ismail Z, Anstey KJ, Mortby M (2018) Association of Alzheimer's genetic loci with mild behavioral impairment. Am J Med Genet B Neuropsychiatr Genet 177, 727-735.

[18] Ismail Z, Aguera-Ortiz L, Brodaty H, Cieslak A, Cummings J, Fischer CE, Gauthier S, Geda YE, Herrmann N, Kanji J, Lanctot KL, Miller DS, Mortby ME, Onyike CU, Rosen- berg PB, Smith EE, Smith GS, Sultzer DL, Lyketsos C, NPS Professional Interest Area of the International Society of to Advance Alzheimer's Research and Treatment (NPS-PIA of ISTAART) (2017) The Mild Behavioral Impairment Checklist (MBI-C): A rating scale for neuropsychiatric symptoms in pre-dementia populations. J Alzheimers Dis 56, 929-938.

[19] Mallo SC, Ismail Z, Pereiro AX, Facal D, Lojo-Seoane C, Campos-Magdaleno M, Juncos-Rabadan O (2019) Assessing mild behavioral impairment with the mild behavioral impairment checklist in people with subjective cognitive decline. Int Psychogeriatr 31, 231-239.

[20] Mallo SC, Ismail Z, Pereiro AX, Facal D, Lojo-Seoane C, Campos-Magdaleno M, Juncos-Rabadan O (2018) Assessing mild behavioral impairment with the mild behavioral impairment-checklist in people with mild cognitive impairment. J Alzheimers Dis 66, 83-95.

[21] Mortby ME, Ismail Z, Anstey KJ (2018) Prevalence estimates of mild behavioral impairment in a population-based sample of pre-dementia states and cognitively healthy older adults. Int Psychogeriatr 30, 221-232.

[22] Sheikh F, Ismail Z, Mortby ME, Barber P, Cieslak A, Fischer K, Granger R, Hogan DB, Mackie A, Maxwell CJ, Menon B, Mueller P, Patry D, Pearson D, Quickfall J, Sajobi T, Tse E, Wang M, Smith EE, PROMPT registry investigators (2018) Prevalence of mild behavioral impairment in mild cognitive impairment and subjective cognitive decline, and its association with caregiver burden. Int Psychogeriatr 30, 233-244.

[23] McKeith IG, Dickson DW, Lowe J, Emre M, O'Brien JT, Feldman H, Cummings J, Duda JE, Lippa C, Perry EK, Aarsland D, Arai H, Ballard CG, Boeve B, Burn DJ, Costa D, Del Ser T, Dubois B, Galasko D, Gauthier S, Goetz CG, Gomez-Tortosa E, Halliday G, Hansen LA, Hardy J, Iwatsubo T, Kalaria RN, Kaufer D, Kenny RA, Korczyn A, Kosaka K, Lee VM, Lees A, Litvan I, Londos E, Lopez OL, Minoshima S, Mizuno Y, Molina JA, Mukaetova-Ladinska EB, Pasquier F, Perry RH, Schulz JB, Trojanowski JQ, Yamada M, Consortium on DLB (2005) Diagnosis and management of dementia with Lewy bodies: Third report of the DLB Consortium. Neurology 65, 1863-1872.

[24] Jessen F, Amariglio RE, van Boxtel M, Breteler M, Ceccaldi M, Chetelat G, Dubois B, Dufouil C, Ellis KA, van der Flier WM, Glodzik L, van Harten AC, de Leon MJ, McHugh P, Mielke MM, Molinuevo JL, Mosconi L, Osorio RS, Perrotin A, Petersen RC, Rabin LA, Rami L, Reisberg B, Rentz DM, Sachdev PS, de la Sayette V, Saykin AJ, Scheltens P, Shulman MB, Slavin MJ, Sperling RA, Stewart $\mathrm{R}$, Uspenskaya O, Vellas B, Visser PJ, Wagner M, Subjective Cognitive Decline Initiative Working Group (2014) A conceptual framework for research on subjective cognitive decline in preclinical Alzheimer's disease. Alzheimers Dement 10, 844-852.

[25] Ismail Z, Elbayoumi H, Fischer CE, Hogan DB, Millikin CP, Schweizer T, Mortby ME, Smith EE, Patten SB, Fiest KM (2017) Prevalence of depression in patients with mild cognitive impairment: A systematic review and meta-analysis. JAMA Psychiatry 74, 58-67.

[26] Acosta I, Borges G, Aguirre-Hernandez R, Sosa AL, Prince M, Dementia Research G (2018) Neuropsychiatric symptoms as risk factors of dementia in a Mexican population: A 10/66 Dementia Research Group study. Alzheimers Dement 14, 271-279.

[27] Ismail Z, Gatchel J, Bateman DR, Barcelos-Ferreira R, Chantillon M, Jaeger J, Donovan NJ, Mortby ME (2018) Affective and emotional dysregulation as pre-dementia risk 
markers: Exploring the mild behavioral impairment symptoms of depression, anxiety, irritability, and euphoria. Int Psychogeriatr 30, 185-196.

[28] Singh-Manoux A, Dugravot A, Fournier A, Abell J, Ebmeier K, Kivimaki M, Sabia S (2017) Trajectories of depressive symptoms before diagnosis of dementia: A 28-year followup study. JAMA Psychiatry 74, 712-718.

[29] Almeida OP, Hankey GJ, Yeap BB, Golledge J, Flicker L (2017) Depression as a modifiable factor to decrease the risk of dementia. Transl Psychiatry 7, e1117.

[30] Hanseeuw BJ, Jonas V, Jackson J, Betensky RA, Rentz DM, Johnson KA, Sperling RA, Donovan NJ (2018) Association of anxiety with subcortical amyloidosis in cognitively normal older adults. Mol Psychiatry, doi: 10.1038/s41380018-0214-2

[31] Cieslak A, Smith EE, Lysack J, Ismail Z (2018) Case series of mild behavioral impairment: Toward an understanding of the early stages of neurodegenerative diseases affecting behavior and cognition. Int Psychogeriatr 30, 273-280.

[32] Jalal H, Ganesh A, Lau R, Lysack J, Ismail Z (2014) Cholinesterase-inhibitor associated mania: A case report and literature review. Can J Neurol Sci 41, 278-280.

[33] Woolley JD, Khan BK, Murthy NK, Miller BL, Rankin KP (2011) The diagnostic challenge of psychiatric symptoms in neurodegenerative disease: Rates of and risk factors for prior psychiatric diagnosis in patients with early neurodegenerative disease. J Clin Psychiatry 72, 126-133.

[34] Harris BS, Kotsopoulos EJ, Yamin S (2014) Phenotypic cognitive impairment in late-onset delusional disorder. Int Psychogeriatr 26, 965-975.
[35] Matsuoka T, Fujimoto H, Kato Y, Fukui K, Narumoto J (2015) Late-onset psychosis in older outpatients: A retrospective chart review. Int Psychogeriatr 27, 694-696.

[36] Jessen F, Wolfsgruber S, Wiese B, Bickel H, Mosch E, Kaduszkiewicz H, Pentzek M, Riedel-Heller SG, Luck T, Fuchs A, Weyerer S, Werle J, van den Bussche H, Scherer M, Maier W, Wagner M, German Study on Aging, Cognition and Dementia in Primary Care Patients (2014) AD dementia risk in late MCI, in early MCI, and in subjective memory impairment. Alzheimers Dement 10, 76-83.

[37] Reisberg B, Gauthier S (2008) Current evidence for subjective cognitive impairment (SCI) as the pre-mild cognitive impairment (MCI) stage of subsequently manifest Alzheimer's disease. Int Psychogeriatr 20, 1-16.

[38] McKeith IG, Boeve BF, Dickson DW, Halliday G, Taylor JP, Weintraub D, Aarsland D, Galvin J, Attems J, Ballard CG, Bayston A, Beach TG, Blanc F, Bohnen N, Bonanni L, Bras J, Brundin P, Burn D, Chen-Plotkin A, Duda JE, ElAgnaf O, Feldman H, Ferman TJ, Ffytche D, Fujishiro H, Galasko D, Goldman JG, Gomperts SN, Graff-Radford NR, Honig LS, Iranzo A, Kantarci K, Kaufer D, Kukull W, Lee VMY, Leverenz JB, Lewis S, Lippa C, Lunde A, Masellis M, Masliah E, McLean P, Mollenhauer B, Montine TJ, Moreno E, Mori E, Murray M, O'Brien JT, Orimo S, Postuma RB, Ramaswamy S, Ross OA, Salmon DP, Singleton A, Taylor A, Thomas A, Tiraboschi P, Toledo JB, Trojanowski JQ, Tsuang D, Walker Z, Yamada M, Kosaka K (2017) Diagnosis and management of dementia with Lewy bodies: Fourth consensus report of the DLB Consortium. Neurology 89, 88-100. 